\title{
Tissue-specific ramp sequences correspond with increased gene expression in humans and SARS- CoV-2
}

Justin Miller

Sanders-Brown Center on Aging, University of Kentucky

Taylor Meurs

Brigham Young University

Matthew Hodgman

Sanders-Brown Center on Aging, University of Kentucky

Benjamin Song

Brigham Young University

Mark Ebbert

Sanders-Brown Center on Aging, University of Kentucky

John Kauwe

Brigham Young University

Perry Ridge ( $\sim$ perry.ridge@byu.edu )

Brigham Young University https://orcid.org/0000-0001-6944-2753

\section{Article}

Keywords: ramp sequence, SARS-CoV-2, translation efficiency, codon usage bias, tissue, cell-type, web interface

Posted Date: August 12th, 2021

DOI: https://doi.org/10.21203/rs.3.rs-738082/v1

License: (a) (1) This work is licensed under a Creative Commons Attribution 4.0 International License. Read Full License 


\section{Abstract}

Translational ramp sequences are essential regulatory elements that have yet to be characterized in specific tissues. Ramp sequences increase gene expression by evenly spacing ribosomes and slowing initial translation. Therefore, the relative codon adaptiveness within different tissues changes the existence of a ramp sequence without altering the underlying genetic code. Here, we present the first comprehensive analysis of tissue and cell type-specific ramp sequences, and report 3,108 genes with ramp sequences that change between tissues and cell types. The Ramp Atlas (https://ramps.byu.edu/) is an accompanying web portal that allows researchers to query ramp sequences in 18,388 genes across 62 tissues and 66 cell types. We also identified seven SARS-CoV-2 genes and seven human SARS-CoV-2 entry factor genes with tissue-specific ramp sequences that may help explain viral proliferation within those tissues. We anticipate that The Ramp Atlas will facilitate future tissue-specific ramp sequence analyses to develop targeted therapeutics for human disease.

\section{Introduction}

Tissue-specific regulation and resource availability directly affect gene transcription and translation, resulting in widespread tissue-specific differential gene expression ${ }^{1-5}$. The Human Protein Atlas ${ }^{6}$, The Genotype-Tissue Expression (GTEx) Project ${ }^{7}$, and The Functional Annotation of Mammalian Genomes (FANTOM5) $^{8,9}$ report gene expression in different tissues and cell types, which can be leveraged to identify disease biomarkers ${ }^{6,10-20}$ for a variety of diseases such as cancers ${ }^{1-5}$, drug resistance ${ }^{18}$, cystic fibrosis ${ }^{14}$, cardiovascular disease ${ }^{16}$, and Alzheimer's disease ${ }^{20}$.

Since codon usage biases correlate with local tRNA pools and tissue-specific RNA binding proteins ${ }^{21,22}$, codon usage biases often differ across different tissues ${ }^{21,23}$. Variations in tRNA abundance affect gene expression by altering tRNA competition and codon optimality, which changes the translational efficiency of various codons ${ }^{24,25}$. Therefore, although the mRNA transcript remains unchanged between tissues, the efficiency at which those codons can be translated differs between tissues and cell types ${ }^{22,26-30}$. While genome-wide codon usage biases are highly correlated with genome-wide guanine-cytosine (GC) content ${ }^{31}$, translational selection may play an important role in maintaining gene-specific codon usage biases $^{32-34}$ because codon usage biases alter translational efficiency ${ }^{32,35,36}$, gene expression ${ }^{35,37,38}$, mRNA secondary structure ${ }^{34,39}$, and protein structure and function ${ }^{36}$. However, selection for tissuespecific codon adaptation in humans has been highly controversial: Doherty and Mclnerney ${ }^{40}$ and Chamary, et al. ${ }^{32}$ argue that tRNA gene families undergo precise regulation to generate anticodon pools that directly correlate with available mRNA, while Pouyet, et al. ${ }^{41}$ concludes that global GC-content biases affect meiotic recombination and preclude tRNA optimization. Translational selection can also significantly shape codon usage biases across vertebrates, even when high GC-content biases are present ${ }^{42}$. Regardless of selection or GC-content biases shaping the underlying tRNA pool within each tissue, gene expression can be significantly impacted by codon choice. 
Often, tissue-specific tRNA levels correlate with the codon usage biases of highly expressed genes, suggesting that tRNA abundances play a role in tissue-specific gene and protein expression ${ }^{21}$. The tRNA pools within tissues and cell types can differ up to tenfold $21,43-50$, and unique tRNA pools cluster into subsets of proliferating and differentiating cells when characterized by cell type ${ }^{30}$. These cell types maintain tRNA levels that match the codon usage biases and increase the expression of genes that promote proliferation or differentiation ${ }^{30}$. Similarly, cellular tRNA levels may dynamically change to increase the expression of certain genes in response to stress. For example, heterologous protein overexpression, diminished growth rate, and translational imbalances trigger tRNA pool readjustments to increase expression of needed genes ${ }^{51-55}$. Additionally, cells significantly increase tRNA levels in the G2 phase of the cell cycle to temporarily increase the translational efficiency and expression of cycleregulated genes with non-optimal codon usage bias ${ }^{56}$.

Various diseases also trigger tRNA pool alterations. For example, the tRNA pool in breast cancer cells is altered to increase expression of genes that promote metastasis ${ }^{57}$. The interplay between tissue and cell type-specific codon usage biases, tissue and cell type-specific tRNA abundances, and synonymous mutations can have a significant effect on various human diseases ${ }^{30,43-45,57-63}$. During the later stages of infection, HIV-1 changes cellular tRNA levels to increase expression of viral genes with codon usage biases that are poorly adapted to the original host tRNA pool ${ }^{64}$. Similarly, viral genes in SARS-CoV-2 alter tRNA levels and deprive tRNA from normally highly-expressed host genes by monopolizing the tRNA supply, thereby decreasing host gene expression and inducing additional damage ${ }^{65}$.

Viruses that infect humans often use similar codon usage biases as highly expressed genes within the tissues that they infect to facilitate co-opting available cellular machinery ${ }^{65-67}$. For example, genes in SARS-CoV-2 utilize codon usage biases that match very highly expressed genes in lung tissue, likely contributing to increased viral gene expression, decreased host gene expression, and infection in the lungs ${ }^{65,68}$. In SARS-CoV-2, codon usage similarities between viral genes and highly-expressed host genes likely improve viral translation and replication, which may help predict which human genes will be downregulated during an infection ${ }^{69}$. Similarly, codon usage biases in Papillomavirus genes promote gene expression in differentiated epithelial cells to more effectively spread infection ${ }^{70}$.

Although many tissue-specific codon usage biases have previously been reported, the tissue-specific effects of ramp sequences have yet to be characterized. Ramp sequences increase overall translational efficiency by utilizing slowly-translated codons at the beginning of genes to evenly space ribosomes and reduce downstream ribosomal collisions. Ramp sequences occur when 20-40 suboptimal codons concentrate at the beginning ( $5^{\prime}$ end) of highly-expressed gene sequences and occur in approximately $10 \%$ of genes ${ }^{71}$. Ramp sequences generally increase gene expression by counterintuitively slowing initial translation so that ribosomes evenly distribute $e^{21,49,71-74}$ and the remaining transcript can be more efficiently translated without ribosomal traffic jams ${ }^{75,76}$. Increased translational efficiency increases mRNA stability and gene and protein expression, especially in genes that have higher ribosome density, 
higher mRNA levels, and a strong correlation between mRNA and protein expression ${ }^{75,77}$. However, the mechanisms by which ramp sequences affect gene expression have yet to be fully characterized, and ramp sequences may also impact transcriptional efficiency by requiring fewer dsDNA bonds to be broken ${ }^{78}$. While variations exist within different human populations ${ }^{79}$, orthologous ramp sequences are evolutionarily conserved across all domains of life ${ }^{80-82}$. However, the extent to which ramp sequences differ between tissues and cell types has never been studied, despite the direct effect that ramp sequences have on gene expression.

Here, we present the Ramp Atlas, which is the first web portal to analyze tissue and cell type-specific ramp sequences. We identified 3,108 genes with ramp sequences that were alternatively present, or absent, depending on the relative translational efficiencies in tissues and tissue-stratified cell types calculated from gene expression in the Human Protein Atlas ${ }^{83-85}$, GTEx Project ${ }^{7}$, and FANTOM5 datasets $^{8,9}$. We allow researchers to query ramp sequences in 18,388 genes spanning 62 tissues and 66 cell types. Embedded Tableau charts allow user-specific querying to facilitate targeted ramp sequence analyses. Additionally, we identify ramp sequences in seven SARS-CoV-2 genes and seven human SARS-CoV-2 entry factor genes, both present in a variety of tissues. The SARS-CoV-2 analyses indicate that virusspecific ramp sequences may play an integral role in viral proliferation. We anticipate that the Ramp Atlas will facilitate future ramp sequence analyses on tissue or cell type-specific gene expression impacted by ramp sequences, genetic variant effects on tissue-specific gene expression, viral adaptations to specific tissues or cell types, and therapeutic developments that aim to modulate tissue-specific gene expression.

\section{Results}

The Ramp Atlas (https://ramps.byu.edu) is a comprehensive online repository of tissue and cell typespecific ramp sequences spanning 18,388 human genes in 62 tissues and 66 cell types. Additional interactive comparisons of SARS-CoV-2 ramp sequences and human entry factors are also included in this database. This resource provides a template for conducting ramp sequence analyses on viruses and identifying ramp sequences that are correlated with tissue or cell type-specific differential gene expression. Interactive graphics enable comprehensive user-specific analyses that can be integrated into a variety of downstream genetic analyses.

The Ramp Atlas contains the first comprehensive analysis of tissue and cell type-specific ramp sequences and their correlation with protein levels. All results can be queried online at The Ramp Atlas. Table 1 shows that the presence of a ramp sequence significantly correlates with higher gene expression

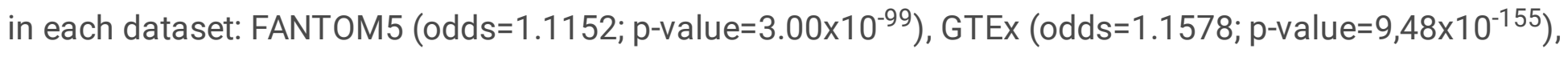
Human Protein Atlas (odds=1.1947; p-value=1.27x10-306), and consensus (odds=1.1477; $p$ -

value $=1.00 \times 10^{-254}$ ). Genes with tissue-specific ramp sequences (i.e., ramp sequences that were present in only some tissues) were also significantly more likely to increase gene expression in the consensus dataset (odds: 1.1937; p-value $=5.56 \times 10^{-5}$ ), FANTOM5 (odds: 1.1314; $p$-value=0.00589), and the Human 
Protein Atlas (odds: 1.1897 ; $p$-value $=2.74 \times 10^{-5}$ ), although the difference was not significant in the GTEx dataset (odds: 0.9242; $\mathrm{p}$-value=0.15236). Notably, GTEx has fewer tissue samples than all other datasets, with only 34 tissues compared to 43 in the Human Protein Atlas, 45 in FANTOM5, and 62 in the consensus dataset.

A chi-squared test shows that ramp sequence prevalence differs significantly across all tissue types in every dataset (FANTOM5 p-value $<2.23 \times 10^{-308}$, GTEx p-value $=8.52 \times 10^{-81}$, Human Protein Atlas pvalue $<2.23 \times 10^{-308}$, consensus $p$-value $<2.23 \times 10^{-308}$, cell type $p$-value $=6.06 \times 10^{-20}$ ). Pairwise two-proportion $z$-tests that correct for multiple testing in each dataset showed that the proportion of genes with ramp sequences also differed significantly in many pairwise tissue comparisons: $43.99 \%$ of tissue comparisons in the consensus dataset, $50.51 \%$ of comparisons in FANTOM5, 35.65\% of comparisons in GTEx, and $62.57 \%$ of comparisons in the Human Protein Atlas. Similarly, pairwise two-proportion z-tests in the Human Protein Atlas cell type dataset show that proportions of ramp sequences differed significantly in $4.34 \%$ of tissue-stratified cell type comparisons after correcting for multiple tests. The proportion of unique ramp sequences in the thymus, rectum, and total peripheral blood mononuclear cells (PBMC) were the highest compared to all other tissues (see Figure 1). We found that all 66 tissuestratified cell types had at least one significant z-test result. Ramp sequence presence is most likely to differ in the seminiferous ducts in testis, cells in the white pulp in the spleen, and glandular cells in the parathyroid gland. Cells in the white pulp in the spleen use fewer ramp sequences than average, while the other two tissue-stratified cell types have more ramp sequences than average (see Figure 2). Figure 3 shows the percentage of ramp sequences shared between each tissue in the consensus dataset. The average percentage of ramp sequences shared between the urinary bladder and all other tissues is the highest (87.46\%), while the rectum has is the fewest overlapping ramp sequences with other tissues (57.26\%). Figure 4 shows ramp sequence overlap between different cell types from the Human Protein Atlas tissue-stratified cell types. Trophoblast cells in the placenta have the highest percentage of shared ramp sequences with other tissue-stratified cell types (94.96\%), while cells in the seminiferous cells in the testes have the lowest $(76.89 \%)$.

The "Search Database" page on The Ramp Atlas contains several interactive Tableau charts that allow users to query 18,388 genes for ramp sequences and explore differences between each dataset. For convenience, users can view ramp sequence results for multiple genes and datasets side-by-side. These interactive Tableau charts also display normalized gene expression levels across tissues, cell types, and datasets. Users can quickly view the extent to which ramp sequences differ between different tissues and visually determine if ramp sequences potentially affect gene expression. Interactive versions of Figures 14 (introduced above) are also available on the "Search Database" page, which enable users to view tissue and cell type differences in ramp sequence presence in more detail. These ramp sequence results are also available for bulk download on the "Downloads" page (https://ramps.byu.edu/Downloads). 
We identified ramp sequences in seven SARS-CoV-2 genes: the 2'-O-RNA methyltransferase, the leader protein (non-structural protein 1), non-structural protein 6 , the nucleocapsid phosphoprotein, the ORF3a protein, the ORF7a Nanoluciferase protein, and the surface glycoprotein. Ramp sequences in all but two of these genes (non-structural protein 6 and the surface glycoprotein) are tissue-specific (see Figure 5). Similarly, seven of the 26 analyzed human genes that encode SARS-CoV-2 entry factors ${ }^{86}$ show tissuespecificity: Transmembrane protease, serine 2 (TMPRSS2); Membrane alanyl aminopeptidase (ANPEP); Charged Multivesicular Body Protein 2A (CHMP2A); Cathepsin L (CTSL); Furin, Paired Basic Amino Acid Cleaving Enzyme (FURIN); RAB1A, Member RAS Oncogene Family (RAB1A); and Transmembrane Anterior Posterior Transformation 1 (TAPT1)(see Figure 6). By combining these two analyses, we ranked tissues by ramp sequence prevalence in both SARS-CoV-2 and human entry factor genes (see Figure 7). A onetailed t-test using these data shows that tissues with high SARS-CoV-2 proliferation have significantly more SARS-CoV-2 and human entry factor genes with ramp sequences than tissues that show less SARSCoV-2 proliferation ( $p$-value $=0.009918$ ). Both the rectum and duodenum (tissues with high SARS-CoV-2 proliferation ${ }^{87}$ ) showed the most unique ramp sequence usage (see Figure 8 ).

Users can interactively query Tableau charts on the SARS-CoV-2 page of The Ramp Atlas to thoroughly explore ramp sequences within the SARS-CoV-2 genome as well as in seven human entry factors for SARS-CoV-2. An interactive heat map at the top of the page shows the percentage of SARS-CoV-2 ramp sequences that are shared between different tissues in the consensus dataset. Below, another interactive chart presents data on the presence of ramp sequences in different SARS-CoV-2 genes and across the 62 tissues in the consensus dataset. Under the "Ramp Sequences in Human Entry Factors" section of the webpage, an interactive Tableau chart presents data for the presence of ramp sequences in SARS-CoV-2 human entry factors across all tissues in the consensus dataset. These interactive charts allow users to thoroughly explore ramp sequences in SARS-CoV-2.

\section{Discussion}

\section{Tissue-Specific Ramp Sequence Regulation}

We show that ramp sequences change based on the relative codon adaptiveness within a specific tissue or cell type. Those changes often result in gene-specific ramp sequences that are unique to a tissue or cell type. Tissue and cell type-specific ramp sequences are highly associated with increased gene expression when a ramp sequence is present. This study is the first comprehensive analysis of variable ramp sequences and presents a framework for conducting future tissue and cell type-specific ramp sequence analyses online. We propose that future ramp sequence calculations should consider ramp sequence 
variability that may occur within an organism based on tissue-specific codon optimality. We also propose that variable ramp sequences might be an additional mechanism for regulating tissue and cell typespecific differential gene expression that warrants further exploration.

\section{SARS-CoV-2 Ramp Sequence Analysis}

Ramp sequences may play a significant role in determining tissue-specific SARS-CoV-2 infection and proliferation because tissues that experience higher rates of SARS-CoV-2 infection have significantly more ramp sequences in SARS-CoV-2 and associated human entry factor genes. These tissue-specific ramp sequences are predicted to increase expression of SARS-CoV-2 genes as well as the human genes that facilitate SARS-CoV-2 entry into host cells, resulting in tissue-specific increased rates of infection and proliferation. Specifically, tissues and tissue-stratified cell types with increased expression of human entry factors ACE2, TMPRSS2, and CTSL show increased SARS-CoV-2 infection ${ }^{87-91}$. While both TMPRSS2 and CTSL contain ramp sequences, only ramp sequences in TMPRSS2 are tissue-specific and therefore may influence which tissues are most infected by SARS-CoV-2. Additionally, tissue-specific ramp sequences in the SARS-CoV-2 leader protein, which inhibits host gene expression ${ }^{92,93}$ and helps the virus escape type-1 interferon cellular responses ${ }^{92-94}$, may also influence tissue-specific infection and proliferation.

In addition to potentially contributing to SARS-CoV-2 tissue-specific infection and proliferation, ramp sequences may also influence other fatal complications commonly found in SARS-CoV-2 patients. SARSCoV-2 genes ORF3 and non-structural protein 1 (NSP1) respectively significantly upregulate and downregulate $A P O B$ expression ${ }^{95}$. Overexpression of $A P O B$ is associated with atherosclerosis ${ }^{96}$, a condition that contributes to the severity of SARS-CoV-2 infection ${ }^{95,97}$. Both ORF3 and NSP1 have ramp sequences specifically in the liver and small intestine, where $A P O B$ expression is highest ${ }^{83,98}$. These ramp sequences may significantly increase $O R F 3$ and $N S P 1$ expression within the liver and small intestine, likely affecting $A P O B$ expression. While $O R F 3$ and $N S P 1$ have opposite effects on $A P O B$ expression, ramp sequences may play an important role in determining the magnitude of those effects, and consequently, the complications of atherosclerosis associated with increased $A P O B$ expression. The Ramp Atlas provides a framework to conduct similar analyses on novel viral strains and viral variants, which may help prioritize tissues and cell types likely to be affected by a virus.

\section{Conclusion}

The Ramp Atlas is the first comprehensive analysis of tissue and cell type-specific ramp sequences. We identified ramp sequences in SARS-CoV-2 that likely contribute to its widespread proliferation, and we show that those ramp sequences are more likely to occur in tissues known to be affected by SARS-CoV-2. Additionally, we provide a template for conducting similar ramp sequence analyses using a web resource to facilitate the widespread adaptation of ramp sequences in a variety of future applications.

The Ramp Atlas will help researchers better understand how ramp sequences affect gene expression, which may lead to more specific, targeted therapeutics. Tissue-specific gene and protein expression can 
affect tissue-specific responses to drug therapies ${ }^{99,100}$. For example, gene and protein expression profiles collected from tumor tissue samples can accurately predict patient responses to different cancer therapies ${ }^{101-106}$. Because ramp sequence usage differs significantly between tissues and tissue-stratified cell types and can be highly correlated with local protein expression, genetic variation affecting ramp sequences may also affect drug response and overall gene expression. Therefore, clinical trials targeting gene expression may need to incorporate the impact of tissue-specific ramp sequences for optimal results. The Ramp Atlas is a hypothesis-generating platform that provides a framework for researchers to incorporate ramp sequences in creative protein regulation and expression analyses.

\section{Methods}

\section{Identifying Ramp Sequences}

Ramp sequences are calculated by identifying statistical outliers of codon efficiencies concentrated at the $5^{\prime}$ end of gene coding sequences using the software package, ExtRamp ${ }^{71}$. Codon efficiencies are measured by the relative synonymous codon usage metric, which ranges from 0-1 and is calculated by dividing the number of occurrences for a specific codon by the number of occurrences for the most common codon that encodes the same amino acid. We used pre-computed relative synonymous codon usage values calculated in April 2021 for 43 tissues in the Human Protein Atlas ${ }^{83-85}, 34$ tissues in the GTEx Project ${ }^{7}, 45$ tissues in the FANTOM 5 datasets $^{8,9}$, and 62 tissues in the consensus dataset found in the Human Protein Atlas, which combines gene expression from all three databases. We also used precomputed synonymous codon usages spanning 66 tissue-stratified cell types, which were also downloaded in April 2021. We used default parameters in ExtRamp to identify ramp sequences in each tissue and cell type for 18,388 genes, where all annotated gene isoforms were required to have similar gene expression (i.e., gene expression was within the same quartile for each isoform). The longest isoform from the GRCh38 reference genome was considered representative for each gene and was used to identify ramp sequences in each tissue or cell type. Ramp sequence identification was performed for each tissue and cell type separately. Two output files were generated for each run: 1) ramp sequences identified by ExtRamp, and 2) genes that did not contain a predicted ramp sequence within the tissue or cell type. The original data files downloaded from the Human Protein Atlas were modified to include an extra column, "Ramp presence," with values of "Ramp" or "No ramp" for each gene in each tissue and cell type. These comma-separated values (CSV) files are available at https://ramps.byu.edu/Downloads. We also present these data in Tableau charts on the "Search Database" page of the Ramp Atlas, which allows users to view tissues where ramp sequences exist for a gene and compare the presence or absence of a ramp sequence to the normalized gene expression for each tissue. 
We performed chi-squared analyses of ramp sequence presence across all datasets and then calculated the odds of both overall and tissue-specific increased expression if a gene contains a ramp sequence.

To determine if genes with ramp sequences tend to be highly expressed, we first grouped all genes across all tissues by whether or not they contained a ramp sequence (e.g., if gene $\mathrm{A}$ had a ramp sequence in 30 tissues and did not have a ramp sequence in 32 tissues, then it would be included 30 times in the "ramp" group and 32 times in the "no ramp" group with the corresponding gene expression for each tissue). We then calculated the odds of having "medium" or "high" expression versus "not detected" or "low" expression for each group. We calculated the relative odds of having increased gene expression with a ramp by dividing the odds of having "medium" or "high" expression in the "ramp" group by the odds of having "medium" or "high" expression in the "no ramp" group.

We performed the following calculations in each dataset to determine the extent to which genes with a ramp sequence in some tissues and not others are differentially expressed. First, we ensured that each gene had a ramp sequence in at least $5 \%$ of the tissues and did not have a ramp sequence at least $5 \%$ of the tissues. The average gene expression in the "ramps" and "no ramps" groups were calculated for each gene separately to limit the potential effects of sampling. The expression bins were transformed to follow a linear progression between "not detected" and "high expression" (i.e., "not detected" was given a weight of "1" and "high expression" was given a weight of "4"), and the average expression for each group (e.g., "ramps" and "no ramps") was calculated as follows:

$$
\text { Average Expression }=\frac{(1 * \text { not detected })+(2 * \text { low })+(3 * \text { medium })+(4 * \text { high })}{\text { total number of sequences }}
$$

Finally, we counted the number of genes that had a higher tissue-specific gene expression when they had a ramp sequence compared to when they did not have a ramp sequence and calculated the odds of tissue-specific variable ramp sequences correlating with increased gene expression (see Table 2).

\section{Comparing Ramp Presence across Tissue and Cell Types}

We compared the proportion of genes with ramp sequences across different tissues or cell types for each dataset (e.g., FANTOM5, GTEx, Human Protein Atlas, consensus, and Human Protein Atlas cell types) and determined if the same genes always contained ramp sequences. 
We conducted a single chi-squared test for each dataset to determine the extent to which ramp sequences are distributed across tissue and cell types. Each tissue contained at least 2,425 ramp sequences (i.e., $14 \%$ of sampled genes) and each cell type contained at least 1,521 ramp sequences (i.e., $15 \%$ of sampled genes), which was a large enough sample size to adequately assess the distribution using the chi-squared test.

We followed these dataset-wide chi-squared tests with more specific pairwise, two-proportion z-tests between each tissue type to identify which tissues had the most unique ramp sequences. We applied a Bonferroni correction for multiple tests (FANTOM5 alpha $=5.05 \times 10^{-5}$, GTEx alpha $=8.91 \times 10^{-5}$, Human Protein Atlas alpha $=5.54 \times 10^{-5}$, consensus alpha $=2.64 \times 10^{-5}$, cell type alpha $\left.=2.33 \times 10^{-5}\right)$. Using Tableau, we created bar charts to display the total number of significant tests for each tissue/cell type, highlighting tissues/cell types with more unique proportions of ramp sequences.

\section{Data Visualization}

We used Tableau Software (www.tableau.com) to create The Ramp Atlas, which generates interactive graphics for users to query ramp sequence data across tissues and cell types. All Tableau charts are available at https://ramps.byu.edu/SearchDatabase. The bulk data files used to generate these Tableau charts are available for download at https://ramps.byu.edu/Downloads.

\section{SARS-CoV-2 Ramp Sequence Analysis}

We downloaded all SARS-CoV-2 nucleotide coding sequences from the National Center for Biotechnology Information (NCBI) SARS-CoV-2 Resources in November 2020. We analyzed 406,882 available SARS-CoV2 gene sequences and overlapping gene bodies. Since SARS-CoV-2 utilizes the host machinery for translation, the optimal codon usage for SARS-CoV-2 should be based on the relative synonymous codon usage of the host tissue. Therefore, we used ExtRamp coupled with the relative synonymous codon usage values calculated from 62 tissue-specific highly expressed genes in the Human Protein Atlas, GTEx, FANTOM5, and consensus datasets to determine the extent to which ramp sequences were present in SARS-CoV-2 genes for each dataset. We then determined the frequency of ramp sequences in SARSCoV-2 genes across each tissue and identified the number of ramp sequences shared between any two tissues. We also identified tissue-specific ramp sequences in human genes that encode SARS-CoV-2 entry factors, as reported in Singh, et al. ${ }^{86}$. We then ranked all tissues according to the total number of ramp sequences present in both SARS-CoV-2 and associated human entry factor genes. Using these data, we performed a one-tailed t-test to compare the mean number of SARS-CoV-2 and human entry factor genes 
with ramp sequences in tissues that show high SARS-CoV-2 proliferation ${ }^{87,107}$ against those tissues that do not show high SARS-CoV-2 proliferation. All analyses are freely available and distributed using an ASP.NET web server hosted at Brigham Young University (https://ramps.byu.edu).

\section{Declarations}

\section{Acknowledgements}

We appreciate the contributions of Brigham Young University (BYU), the Office of Research Computing at BYU, Life Sciences IT at BYU, and the Sanders-Brown Center on Aging at the University of Kentucky for supporting this research. This work was partially funded by the BrightFocus Foundation and its donors through grant \# A2020118F (PI: Miller).

\section{Tables}

Table 1: Odds of Ramp Sequence Presence Correlating with Increased Gene Expression

\begin{tabular}{|c|c|c|c|}
\hline Dataset & Chi-square & P-value & Odds Ratio \\
\hline FANTOM5 & 459.3981 & $3.00 \times 10^{-99}$ & 1.1152 \\
\hline GTEx & 715.4278 & $9.48 \times 10^{-155}$ & 1.1578 \\
\hline Human Protein Atlas & 1415.5104 & $1.27 \times 10^{-306}$ & 1.1947 \\
\hline Consensus & 1176.3328 & $1.00 \times 10^{-254}$ & 1.1477 \\
\hline
\end{tabular}

For each dataset, the chi-squared value and p-value for increased expression if a gene has a ramp sequence as well as the odds of having higher expression if ramp sequence present vs not present. The odds ratio shows a significant difference in gene expression if a gene has a ramp sequence.

Table 2: Odds of Ramp Sequence Presence Correlating with Tissue-Specific Gene Expression 


\begin{tabular}{|c|c|c|c|c|c|}
\hline Dataset & $\begin{array}{c}\text { Genes with Decreased Tissue } \\
\text { Expression }\end{array}$ & $\begin{array}{c}\text { Genes with Increased Tissue } \\
\text { Expression }\end{array}$ & $\begin{array}{c}\text { Chi- } \\
\text { square }\end{array}$ & P-value & $\begin{array}{c}\text { Odds } \\
\text { Ratio }\end{array}$ \\
\hline FANTOM5 & 936 & 1059 & 7.583 & 0.00589 & 1.1314 \\
\hline GTEx & 686 & 634 & 2.0485 & 0.15236 & 0.9242 \\
\hline $\begin{array}{c}\text { Human } \\
\text { Protein Atlas }\end{array}$ & 1070 & 1273 & 17.5881 & $2.74 \times 10^{-5}$ & 1.1897 \\
\hline Consensus & 950 & 1134 & 16.2457 & $5.56 \times 10^{-5}$ & 1.1937 \\
\hline
\end{tabular}

For each dataset, the chi-squared value and p-value for expression if a gene has a ramp sequence as well as the odds of having higher expression in a tissue where a ramp sequence is present. This odds ratio shows a significant difference in tissue-specific gene expression if a gene has a ramp sequence in that specific tissue.

\section{Works Cited}

1 Kosti, I., Jain, N., Aran, D., Butte, A. J. \& Sirota, M. Cross-tissue Analysis of Gene and Protein Expression in Normal and Cancer Tissues. Sci Rep 6, 24799, doi:10.1038/srep24799 (2016).

2 Pontén, F. et al. A global view of protein expression in human cells, tissues, and organs. Mol Syst Bio/ 5, 337, doi:10.1038/msb.2009.93 (2009).

3 Fagerberg, L. et al. Analysis of the human tissue-specific expression by genome-wide integration of transcriptomics and antibody-based proteomics. Mol Cell Proteomics 13, 397-406, doi:10.1074/mcp.M113.035600 (2014).

4 Natarajan, A., Yardimci, G. G., Sheffield, N. C., Crawford, G. E. \& Ohler, U. Predicting cell-type-specific gene expression from regions of open chromatin. Genome Res 22, 1711-1722, doi:10.1101/gr.135129.111 (2012).

$5 \quad$ Kotliar, D. et al. Identifying gene expression programs of cell-type identity and cellular activity with single-cell RNA-Seq. Elife 8, doi:10.7554/eLife.43803 (2019).

6 Uhlén, M. et al. Proteomics. Tissue-based map of the human proteome. Science 347, 1260419, doi:10.1126/science.1260419 (2015).

7 Consortium, G. Human genomics. The Genotype-Tissue Expression (GTEx) pilot analysis: multitissue gene regulation in humans. Science 348, 648-660, doi:10.1126/science.1262110 (2015).

8 Lizio, M. et al. Update of the FANTOM web resource: expansion to provide additional transcriptome atlases. Nucleic Acids Res 47, D752-D758, doi:10.1093/nar/gky1099 (2019). 
9 Lizio, M. et al. Gateways to the FANTOM5 promoter level mammalian expression atlas. Genome Bio/ 16, 22, doi:10.1186/s13059-014-0560-6 (2015).

10 Prassas, I., Chrystoja, C. C., Makawita, S. \& Diamandis, E. P. Bioinformatic identification of proteins with tissue-specific expression for biomarker discovery. BMC Med 10, 39, doi:10.1186/1741-7015-10-39 (2012).

11 Pontén, F., Schwenk, J. M., Asplund, A. \& Edqvist, P. H. The Human Protein Atlas as a proteomic resource for biomarker discovery. J Intern Med 270, 428-446, doi:10.1111/j.1365-2796.2011.02427.x (2011).

12 Uhlén, M. et al. A human protein atlas for normal and cancer tissues based on antibody proteomics. Mol Cell Proteomics 4, 1920-1932, doi:10.1074/mcp.M500279-MCP200 (2005).

13 Mathivanan, S. et al. Proteomics analysis of A33 immunoaffinity-purified exosomes released from the human colon tumor cell line LIM1215 reveals a tissue-specific protein signature. Mol Cell Proteomics 9, 197-208, doi:10.1074/mcp.M900152-MCP200 (2010).

14 Kälin, N., Claass, A., Sommer, M., Puchelle, E. \& Tümmler, B. DeltaF508 CFTR protein expression in tissues from patients with cystic fibrosis. J Clin Invest 103, 1379-1389, doi:10.1172/JCl5731 (1999).

15 Björling, E. et al. A web-based tool for in silico biomarker discovery based on tissue-specific protein profiles in normal and cancer tissues. Mol Cell Proteomics 7, 825-844, doi:10.1074/mcp.M700411MCP200 (2008).

16 Arrell, D. K., Neverova, I. \& Van Eyk, J. E. Cardiovascular proteomics: evolution and potential. Circ Res 88, 763-773, doi:10.1161/hh0801.090193 (2001).

17 Korfali, N. et al. The nuclear envelope proteome differs notably between tissues. Nucleus 3, 552564, doi:10.4161/nucl.22257 (2012).

18 Johnson, B. M., Zhang, P., Schuetz, J. D. \& Brouwer, K. L. Characterization of transport protein expression in multidrug resistance-associated protein (Mrp) 2-deficient rats. Drug Metab Dispos 34, 556562, doi:10.1124/dmd.105.005793 (2006).

19 Rho, J. H., Roehrl, M. H. \& Wang, J. Y. Tissue proteomics reveals differential and compartmentspecific expression of the homologs transgelin and transgelin-2 in lung adenocarcinoma and its stroma. J Proteome Res 8, 5610-5618, doi:10.1021/pr900705r (2009).

20 Smith, M. Z., Nagy, Z. \& Esiri, M. M. Cell cycle-related protein expression in vascular dementia and Alzheimer's disease. Neurosci Lett 271, 45-48, doi:10.1016/s0304-3940(99)00509-1 (1999).

21 Dittmar, K. A., Goodenbour, J. M. \& Pan, T. Tissue-specific differences in human transfer RNA expression. PLoS Genet 2, e221, doi:10.1371/journal.pgen.0020221 (2006). 
22 Payne, B. L. \& Alvarez-Ponce, D. Codon Usage Differences among Genes Expressed in Different Tissues of Drosophila melanogaster. Genome Biol Evol 11, 1054-1065, doi:10.1093/gbe/evz051 (2019).

23 Kames, J. et al. TissueCoCoPUTs: Novel Human Tissue-Specific Codon and Codon-Pair Usage Tables Based on Differential Tissue Gene Expression. J Mol Biol 432, 3369-3378, doi:10.1016/j.jmb.2020.01.011 (2020).

24 Tuller, T. et al. An evolutionarily conserved mechanism for controlling the efficiency of protein translation. Cell 141, 344-354, doi:10.1016/j.cell.2010.03.031 (2010).

25 Shao, Z. Q., Zhang, Y. M., Feng, X. Y., Wang, B. \& Chen, J. Q. Synonymous codon ordering: a subtle but prevalent strategy of bacteria to improve translational efficiency. PLoS One 7, e33547, doi:10.1371/journal.pone.0033547 (2012).

26 Liu, Q. Mutational bias and translational selection shaping the codon usage pattern of tissuespecific genes in rice. PLoS One 7, e48295, doi:10.1371/journal.pone.0048295 (2012).

27 Camiolo, S., Farina, L. \& Porceddu, A. The relation of codon bias to tissue-specific gene expression in Arabidopsis thaliana. Genetics 192, 641-649, doi:10.1534/genetics.112.143677 (2012).

28 Plotkin, J. B., Robins, H. \& Levine, A. J. Tissue-specific codon usage and the expression of human genes. Proc Natl Acad Sci U S A 101, 12588-12591, doi:10.1073/pnas.0404957101 (2004).

29 Sémon, M., Lobry, J. R. \& Duret, L. No evidence for tissue-specific adaptation of synonymous codon usage in humans. Mol Biol Evol 23, 523-529, doi:10.1093/molbev/msj053 (2006).

30 Gingold, $\mathrm{H}$. et al. A dual program for translation regulation in cellular proliferation and differentiation. Cel/ 158, 1281-1292, doi:10.1016/j.cell.2014.08.011 (2014).

31 Zhou, H.-Q., Ning, L.-W., Zhang, H.-X. \& Guo, F.-B. Analysis of the Relationship between Genomic GC Content and Patterns of Base Usage, Codon Usage and Amino Acid Usage in Prokaryotes: Similar GC Content Adopts Similar Compositional Frequencies Regardless of the Phylogenetic Lineages. PLOS ONE 9, e107319, doi:10.1371/journal.pone.0107319 (2014).

32 Chamary, J. V., Parmley, J. L. \& Hurst, L. D. Hearing silence: non-neutral evolution at synonymous sites in mammals. Nat Rev Genet 7, 98-108, doi:10.1038/nrg1770 (2006).

33 dos Reis, M., Savva, R. \& Wernisch, L. Solving the riddle of codon usage preferences: a test for translational selection. Nucleic Acids Res 32, 5036-5044, doi:10.1093/nar/gkh834 (2004).

34 Hia, F. et al. Codon bias confers stability to human mRNAs. EMBO Rep 20, e48220, doi:10.15252/embr.201948220 (2019). 
35 Quax, T. E., Claassens, N. J., Soll, D. \& van der Oost, J. Codon Bias as a Means to Fine-Tune Gene Expression. Mol Cell 59, 149-161, doi:10.1016/j.molcel.2015.05.035 (2015).

36 Zhao, F., Yu, C. H. \& Liu, Y. Codon usage regulates protein structure and function by affecting translation elongation speed in Drosophila cells. Nucleic Acids Res 45, 8484-8492, doi:10.1093/nar/gkx501 (2017).

37 Duret, L. tRNA gene number and codon usage in the C. elegans genome are co-adapted for optimal translation of highly expressed genes. Trends Genet 16, 287-289, doi:10.1016/s0168-9525(00)02041-2 (2000).

38 Duret, L. \& Mouchiroud, D. Expression pattern and, surprisingly, gene length shape codon usage in Caenorhabditis, Drosophila, and Arabidopsis. Proc Natl Acad Sci U S A 96, 4482-4487, doi:10.1073/pnas.96.8.4482 (1999).

39 Chamary, J. V. \& Hurst, L. D. Evidence for selection on synonymous mutations affecting stability of mRNA secondary structure in mammals. Genome Bio/ 6, R75, doi:10.1186/gb-2005-6-9-r75 (2005).

40 Doherty, A. \& Mclnerney, J. O. Translational selection frequently overcomes genetic drift in shaping synonymous codon usage patterns in vertebrates. Mol Biol Evol 30, 2263-2267, doi:10.1093/molbev/mst128 (2013).

41 Pouyet, F., Mouchiroud, D., Duret, L. \& Sémon, M. Recombination, meiotic expression and human codon usage. Elife 6, e27344 (2017).

42 (!!! INVALID CITATION !!! 40,41).

43 Kirchner, S. et al. Alteration of protein function by a silent polymorphism linked to tRNA abundance. PLoS Biol 15, e2000779, doi:10.1371/journal.pbio.2000779 (2017).

44 Pavon-Eternod, M. et al. tRNA over-expression in breast cancer and functional consequences. Nucleic Acids Res 37, 7268-7280, doi:10.1093/nar/gkp787 (2009).

45 Kirchner, S. \& Ignatova, Z. Emerging roles of tRNA in adaptive translation, signalling dynamics and disease. Nat Rev Genet 16, 98-112, doi:10.1038/nrg3861 (2015).

46 Chittum, H. S. et al. Replenishment of selenium deficient rats with selenium results in redistribution of the selenocysteine tRNA population in a tissue specific manner. Biochim Biophys Acta 1359, 25-34, doi:10.1016/s0167-4889(97)00092-x (1997).

47 Sagi, D. et al. Tissue- and Time-Specific Expression of Otherwise Identical tRNA Genes. PLoS Genet 12, e1006264, doi:10.1371/journal.pgen.1006264 (2016). 
48 Kondo, K., Makovec, B., Waterston, R. H. \& Hodgkin, J. Genetic and molecular analysis of eight tRNA(Trp) amber suppressors in Caenorhabditis elegans. J Mol Bio/ 215, 7-19, doi:10.1016/s00222836(05)80090-7 (1990).

49 Waldman, Y. Y., Tuller, T., Shlomi, T., Sharan, R. \& Ruppin, E. Translation efficiency in humans: tissue specificity, global optimization and differences between developmental stages. Nucleic Acids Res 38, 2964-2974, doi:10.1093/nar/gkq009 (2010).

50 Smith, D. W. Problems of translating heterologous genes in expression systems: the role of tRNA. Biotechnol Prog 12, 417-422, doi:10.1021/bp950056a (1996).

51 Gingold, H., Dahan, O. \& Pilpel, Y. Dynamic changes in translational efficiency are deduced from codon usage of the transcriptome. Nucleic Acids Res 40, 10053-10063, doi:10.1093/nar/gks772 (2012).

52 Torrent, M., Chalancon, G., de Groot, N. S., Wuster, A. \& Madan Babu, M. Cells alter their tRNA abundance to selectively regulate protein synthesis during stress conditions. Sci Signa/11, doi:10.1126/scisignal.aat6409 (2018).

53 Pang, Y. L., Abo, R., Levine, S. S. \& Dedon, P. C. Diverse cell stresses induce unique patterns of tRNA up- and down-regulation: tRNA-seq for quantifying changes in tRNA copy number. Nucleic Acids Res 42, e170, doi:10.1093/nar/gku945 (2014).

54 Puri, P. et al. Systematic identification of tRNAome and its dynamics in Lactococcus lactis. Mol Microbio/ 93, 944-956, doi:10.1111/mmi.12710 (2014).

55 Yona, A. H. et al. tRNA genes rapidly change in evolution to meet novel translational demands. Elife 2, e01339, doi:10.7554/eLife.01339 (2013).

56 Frenkel-Morgenstern, M. et al. Genes adopt non-optimal codon usage to generate cell cycledependent oscillations in protein levels. Mol Syst Bio/ 8, 572, doi:10.1038/msb.2012.3 (2012).

57 Goodarzi, H. et al. Modulated Expression of Specific tRNAs Drives Gene Expression and Cancer Progression. Cel/ 165, 1416-1427, doi:10.1016/j.cell.2016.05.046 (2016).

58 Polte, C. et al. Assessing cell-specific effects of genetic variations using tRNA microarrays. BMC Genomics 20, 549, doi:10.1186/s12864-019-5864-1 (2019).

59 Torres, A. G., Batlle, E. \& Ribas de Pouplana, L. Role of tRNA modifications in human diseases. Trends Mol Med 20, 306-314, doi:10.1016/j.molmed.2014.01.008 (2014).

60 Mahlab, S., Tuller, T. \& Linial, M. Conservation of the relative tRNA composition in healthy and cancerous tissues. Rna 18, 640-652, doi:10.1261/rna.030775.111 (2012). 
61 Rudorf, S. Efficiency of protein synthesis inhibition depends on tRNA and codon compositions. PLoS Comput Biol 15, e1006979, doi:10.1371/journal.pcbi.1006979 (2019).

62 Rudolph, K. L. et al. Codon-Driven Translational Efficiency Is Stable across Diverse Mammalian Cell States. PLoS Genet 12, e1006024, doi:10.1371/journal.pgen.1006024 (2016).

63 Pavon-Eternod, M., Gomes, S., Rosner, M. R. \& Pan, T. Overexpression of initiator methionine tRNA leads to global reprogramming of tRNA expression and increased proliferation in human epithelial cells. RNA 19, 461-466, doi:10.1261/rna.037507.112 (2013).

64 van Weringh, A. et al. HIV-1 modulates the tRNA pool to improve translation efficiency. Mol Biol Evol 28, 1827-1834, doi:10.1093/molbev/msr005 (2011).

65 Alonso, A. M. \& Diambra, L. SARS-CoV-2 Codon Usage Bias Downregulates Host Expressed Genes With Similar Codon Usage. Front Cell Dev Bio/ 8, 831, doi:10.3389/fcell.2020.00831 (2020).

66 Chen, F. et al. Dissimilation of synonymous codon usage bias in virus-host coevolution due to translational selection. Nat Ecol Evol 4, 589-600, doi:10.1038/s41559-020-1124-7 (2020).

67 Miller, J. B., Hippen, A. A., Wright, S. M., Morris, C. \& Ridge, P. G. Human viruses have codon usage biases that match highly expressed proteins in the tissues they infect. Biomedical Genetics and Genomics 2, doi:10.15761/bgg.1000134 (2017).

$68 \mathrm{Li}, \mathrm{Y}$. et al. GC usage of SARS-CoV-2 genes might adapt to the environment of human lung expressed genes. Mol Genet Genomics, doi:10.1007/s00438-020-01719-0 (2020).

69 Maldonado, L. L., Bertelli, A. M. \& Kamenetzky, L. Molecular features similarities between SARSCoV-2, SARS, MERS and key human genes could favour the viral infections and trigger collateral effects. Scientific Reports 11, 4108, doi:10.1038/s41598-021-83595-1 (2021).

70 Zhou, J., Liu, W. J., Peng, S. W., Sun, X. Y. \& Frazer, I. Papillomavirus capsid protein expression level depends on the match between codon usage and tRNA availability. $J$ Virol 73, 4972-4982, doi:10.1128/JVI.73.6.4972-4982.1999 (1999).

71 Miller, J. B., Brase, L. R. \& Ridge, P. G. ExtRamp: a novel algorithm for extracting the ramp sequence based on the tRNA adaptation index or relative codon adaptiveness. Nucleic Acids Res, doi:10.1093/nar/gky1193 (2019).

72 Tuller, T. \& Zur, H. Multiple roles of the coding sequence $5^{\prime}$ end in gene expression regulation. Nucleic Acids Res 43, 13-28, doi:10.1093/nar/gku1313 (2015).

73 Verma, M. et al. A short translational ramp determines the efficiency of protein synthesis. Nature Communications 10, 5774, doi:10.1038/s41467-019-13810-1 (2019). 
74 Tuller, T. et al. Composite effects of gene determinants on the translation speed and density of ribosomes. Genome Bio/ 12, R110, doi:10.1186/gb-2011-12-11-r110 (2011).

75 Tuller, T. et al. An Evolutionarily Conserved Mechanism for Controlling the Efficiency of Protein Translation. Cell 141, 344-354, doi:10.1016/j.cell.2010.03.031 (2010).

76 Dana, A. \& Tuller, T. The effect of tRNA levels on decoding times of mRNA codons. Nucleic Acids Res 42, 9171-9181, doi:10.1093/nar/gku646 (2014).

77 Park, H. \& Subramaniam, A. R. Inverted translational control of eukaryotic gene expression by ribosome collisions. PLoS Bio/ 17, e3000396, doi:10.1371/journal.pbio.3000396 (2019).

78 Villada, J. C., Duran, M. F. \& Lee, P. K. Interplay between Position-Dependent Codon Usage Bias and Hydrogen Bonding at the 5区 End of ORFeomes. Msystems 5 (2020).

79 Hodgman, M. W., Miller, J. B., Meurs, T. E. \& Kauwe, J. S. K. CUBAP: an interactive web portal for analyzing codon usage biases across populations. Nucleic Acids Research 48, 11030-11039, doi:10.1093/nar/gkaa863 (2020).

80 Quax, T. E. F., Claassens, N. J., Soll, D. \& van der Oost, J. Codon Bias as a Means to Fine-Tune Gene Expression. Mol. Cell 59, 149-161, doi:10.1016/j.molcel.2015.05.035 (2015).

81 Goodman, D. B., Church, G. M. \& Kosuri, S. Causes and effects of N-terminal codon bias in bacterial genes. Science 342, 475-479, doi:10.1126/science.1241934 (2013).

82 Gorochowski, T. E., Ignatova, Z., Bovenberg, R. A. \& Roubos, J. A. Trade-offs between tRNA abundance and mRNA secondary structure support smoothing of translation elongation rate. Nucleic Acids Res 43, 3022-3032, doi:10.1093/nar/gkv199 (2015).

83 Thul, P. J. \& Lindskog, C. The human protein atlas: A spatial map of the human proteome. Protein Sci. 27, 233-244, doi:10.1002/pro.3307 (2018).

84 Uhlén, M. et al. Tissue-based map of the human proteome. Science 347, 1260419, doi:10.1126/science.1260419 (2015).

85 Ponten, F., Schwenk, J. M., Asplund, A. \& Edqvist, P. H. D. The Human Protein Atlas as a proteomic resource for biomarker discovery. J. Intern. Med. 270, 428-446, doi:10.1111/j.1365-2796.2011.02427.x (2011).

86 Singh, M., Bansal, V. \& Feschotte, C. A Single-Cell RNA Expression Map of Human Coronavirus Entry Factors. Cell Rep 32, 108175, doi:10.1016/j.celrep.2020.108175 (2020).

87 Trypsteen, W., Van Cleemput, J., Snippenberg, W. V., Gerlo, S. \& Vandekerckhove, L. On the whereabouts of SARS-CoV-2 in the human body: A systematic review. PLoS Pathog 16, e1009037, 
doi:10.1371/journal.ppat.1009037 (2020).

88 Muus, C. et al. Single-cell meta-analysis of SARS-CoV-2 entry genes across tissues and demographics. Nat Med 27, 546-559, doi:10.1038/s41591-020-01227-z (2021).

89 Matsuyama, S. et al. Efficient activation of the severe acute respiratory syndrome coronavirus spike protein by the transmembrane protease TMPRSS2. J Viro/ 84, 12658-12664, doi:10.1128/JVI.01542-10 (2010).

90 Glowacka, l. et al. Evidence that TMPRSS2 activates the severe acute respiratory syndrome coronavirus spike protein for membrane fusion and reduces viral control by the humoral immune response. J Viro/ 85, 4122-4134, doi:10.1128/JVI.02232-10 (2011).

91 Hoffmann, M. et al. SARS-CoV-2 Cell Entry Depends on ACE2 and TMPRSS2 and Is Blocked by a Clinically Proven Protease Inhibitor. Cel/ 181, 271-280.e278, doi:10.1016/j.cell.2020.02.052 (2020).

92 Narayanan, K. et al. Severe acute respiratory syndrome coronavirus nsp1 suppresses host gene expression, including that of type I interferon, in infected cells. J Viro/ 82, 4471-4479, doi:10.1128/JVI.02472-07 (2008).

93 Kamitani, W., Huang, C., Narayanan, K., Lokugamage, K. G. \& Makino, S. A two-pronged strategy to suppress host protein synthesis by SARS coronavirus Nsp1 protein. Nat Struct Mol Biol 16, 1134-1140, doi:10.1038/nsmb.1680 (2009).

94 Xia, H. et al. Evasion of Type I Interferon by SARS-CoV-2. Cell Rep 33, 108234, doi:10.1016/j.celrep.2020.108234 (2020).

95 Stukalov, A. et al. Multilevel proteomics reveals host perturbations by SARS-CoV-2 and SARS-CoV. Nature, doi:10.1038/s41586-021-03493-4 (2021).

96 Khalil, M. F., Wagner, W. D. \& Goldberg, I. J. Molecular interactions leading to lipoprotein retention and the initiation of atherosclerosis. Arterioscler Thromb Vasc Bio/ 24, 2211-2218, doi:10.1161/01.ATV.0000147163.54024.70 (2004).

97 Nicolai, L. et al. Immunothrombotic Dysregulation in COVID-19 Pneumonia Is Associated With Respiratory Failure and Coagulopathy. Circulation 142, 1176-1189, doi:10.1161/CIRCULATIONAHA.120.048488 (2020).

98 Demmer, L. A. et al. Tissue-specific expression and developmental regulation of the rat apolipoprotein B gene. Proc Natl Acad Sci U S A 83, 8102-8106, doi:10.1073/pnas.83.21.8102 (1986).

99 Duffy, Á. et al. Tissue-specific genetic features inform prediction of drug side effects in clinical trials. Sci Adv 6, doi:10.1126/sciadv.abb6242 (2020). 
100 Hao, Y., Quinnies, K., Realubit, R., Karan, C. \& Tatonetti, N. P. Tissue-Specific Analysis of Pharmacological Pathways. CPT Pharmacometrics Syst Pharmacol 7, 453-463, doi:10.1002/psp4.12305 (2018).

101 Nevins, J. R. et al. Towards integrated clinico-genomic models for personalized medicine: combining gene expression signatures and clinical factors in breast cancer outcomes prediction. Hum Mol Genet 12 Spec No 2, R153-157, doi:10.1093/hmg/ddg287 (2003).

102 Gordon, G. J. et al. Using gene expression ratios to predict outcome among patients with mesothelioma. J Natl Cancer Inst 95, 598-605, doi:10.1093/jnci/95.8.598 (2003).

103 Nutt, C. L. et al. Gene expression-based classification of malignant gliomas correlates better with survival than histological classification. Cancer Res 63, 1602-1607 (2003).

104 van de Vijver, M. J. et al. A gene-expression signature as a predictor of survival in breast cancer. $N$ Engl J Med 347, 1999-2009, doi:10.1056/NEJMoa021967 (2002).

105 van 't Veer, L. J. et al. Gene expression profiling predicts clinical outcome of breast cancer. Nature 415, 530-536, doi:10.1038/415530a (2002).

106 Jacquemier, J. et al. Protein expression profiling identifies subclasses of breast cancer and predicts prognosis. Cancer Res 65, 767-779 (2005).

107 Deinhardt-Emmer, S. et al. Early postmortem mapping of SARS-CoV-2 RNA in patients with COVID19 and the correlation with tissue damage. Elife 10, doi:10.7554/eLife.60361 (2021).

\section{Figures}


Tissue Ramp Chi-Square Pairs

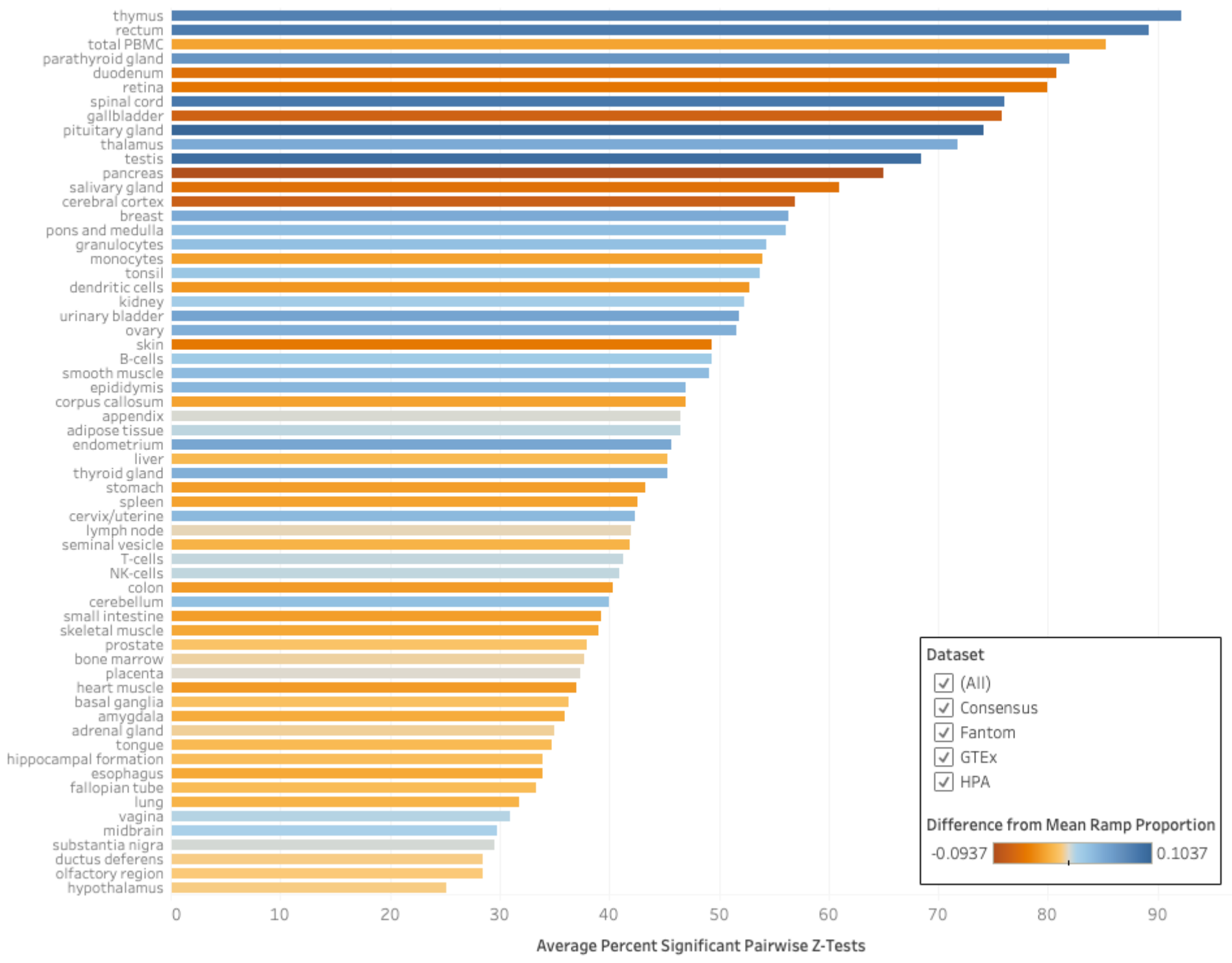

\section{Figure 1}

Percentage of Significant Chi-Square Pairs in Tissue Types. We performed chi-square pair comparisons of ramp sequences across all tissues in each dataset and then pairwise two-proportion z-tests to identify which tissues have the most unique proportions of ramp sequences per gene. This figure shows, for each tissue, the percentage of pairwise z-tests that are significant, averaged across all datasets. Each tissue bar is colored based on the difference between the proportion of genes with ramp sequences and the average proportion of genes with ramp sequences across all tissues. Blue colored tissues have more ramp sequences than average while red colored tissues have fewer ramp sequences than average. 


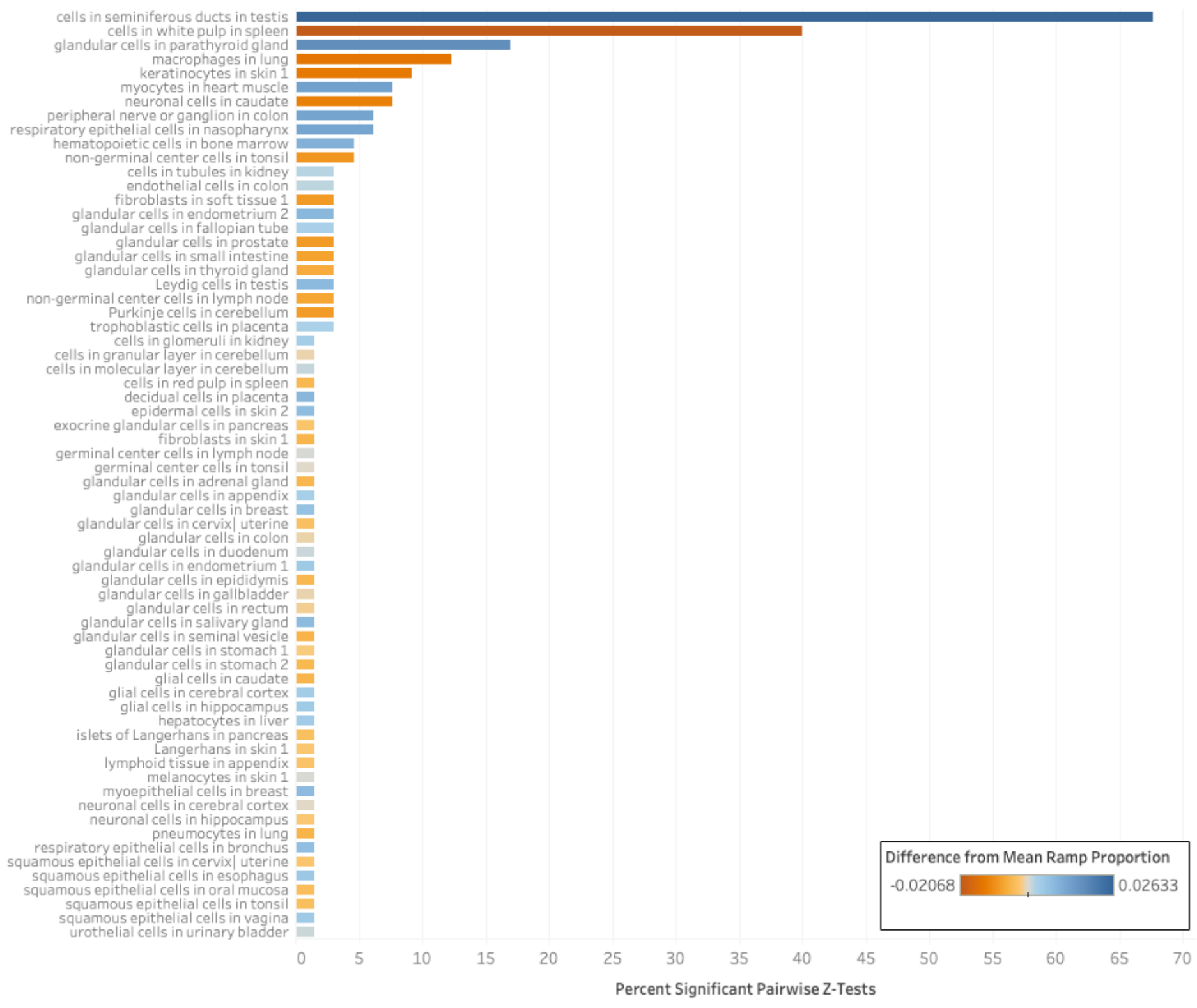

\section{Figure 2}

Percentage of Significant Chi-Square Pairs in Tissue-Stratified Cell Types. We performed chi-square pair comparisons of ramp sequences across all tissue-stratified cell types in each dataset and then pairwise two-proportion z-tests to identify which cell types have the most unique proportions of ramp sequences per gene. This figure shows, for each cell type, the percentage of pairwise z-tests that are significant, averaged across all datasets. Each tissue-stratified cell type bar is colored based on the difference between the proportion of genes with ramp sequences in the cell type and the average proportion of genes with ramp sequences across all cell types. Blue colored tissue-stratified cell types have more ramp sequences than average while red colored cell types have fewer ramp sequences than average. 

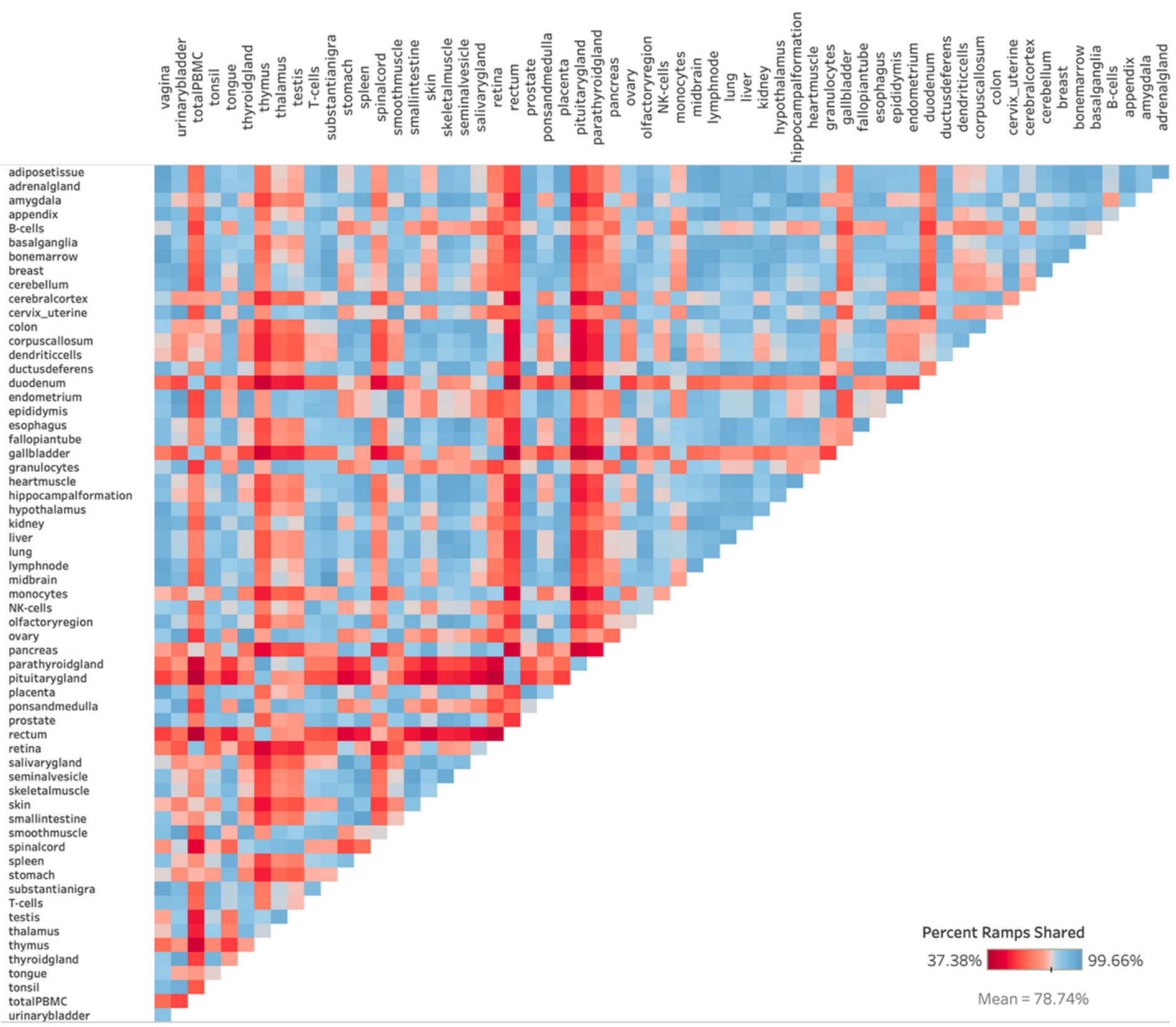

Figure 3

Heat Map of Percent of Ramp Sequences Shared between Tissues. The percent of ramp sequences present in one tissue that are also present in another in the consensus dataset. Red indicates fewer shared ramp sequences and thus a more tissue-specific usage of ramp sequences. Tissue comparisons that are blue have more shared ramp sequences. 
Shared Ramps (HPA Cell Types)

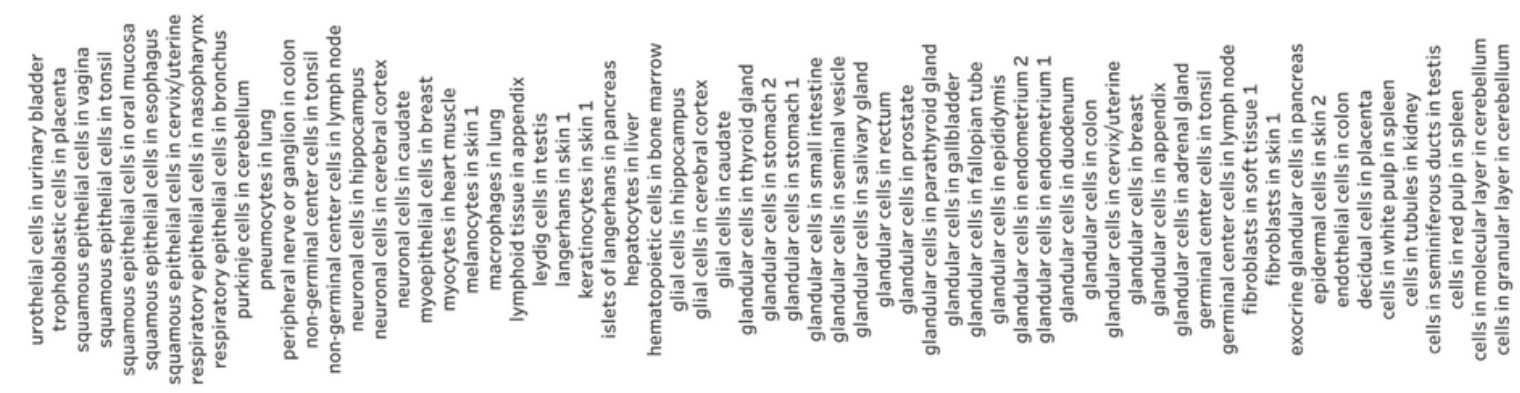

cells in glomeruli in kidney

cells in granular layer in cerebellum

cells in red pulp in spleen

cells in seminiferous ducts

cells in tubules in kidney

decidual cells in placenta

endothelial cells in colo

piderine cells in skin 2 in pancreas

fibroblasts in skin 1

fibroblasts in soft tissue 1

germinal center cells in lymph node

germinal center cells in tonsil

glandular cells in adrenal gland

glandular cells in append

glandular cells in cervix/uterine

glandular cells in colon

glandular cells in duodenum

glandular cells in endometrium 1

glandular cells in endometrium 2

glandular cells in epididymis

landular cells in galopian tube

glandular cells in parathyroid gland

glandular cells in prostate

landular cells in rectum

glandular cells in salivary gland

glandular cells in seminal vesicle

anduar cels in stomact

glandular cells is stomach

glandular cells in thyroid gland

lial cells in caudate

glial cells in cerebral cortex

gilal cells in hippocampus

a marrow

epatocytes in liver

eratinocytes in skin 1 pancreas

angerhans in skin 1

ymphoid tissue in appendix

macrophages in lung

myocytes in heart muscle

myoepithelial cells in breast

euronal cells in caudate

neuronal cells in cerebral cortex

neuronal cells in hippocampus

norminal center cells in lymph node

peripheral nerve or ganglion in colon

pneumocytes in lung

purkinje cells in cerebellum

respiratory epithelial cells in bronchus

espiratory epithelial cells in nasopharynx

Percent Ramps Shared

squamous epithelialcells in cervix/uterine

squamous epithelial cells in oraphus

squamous epithelial cells in tonsil

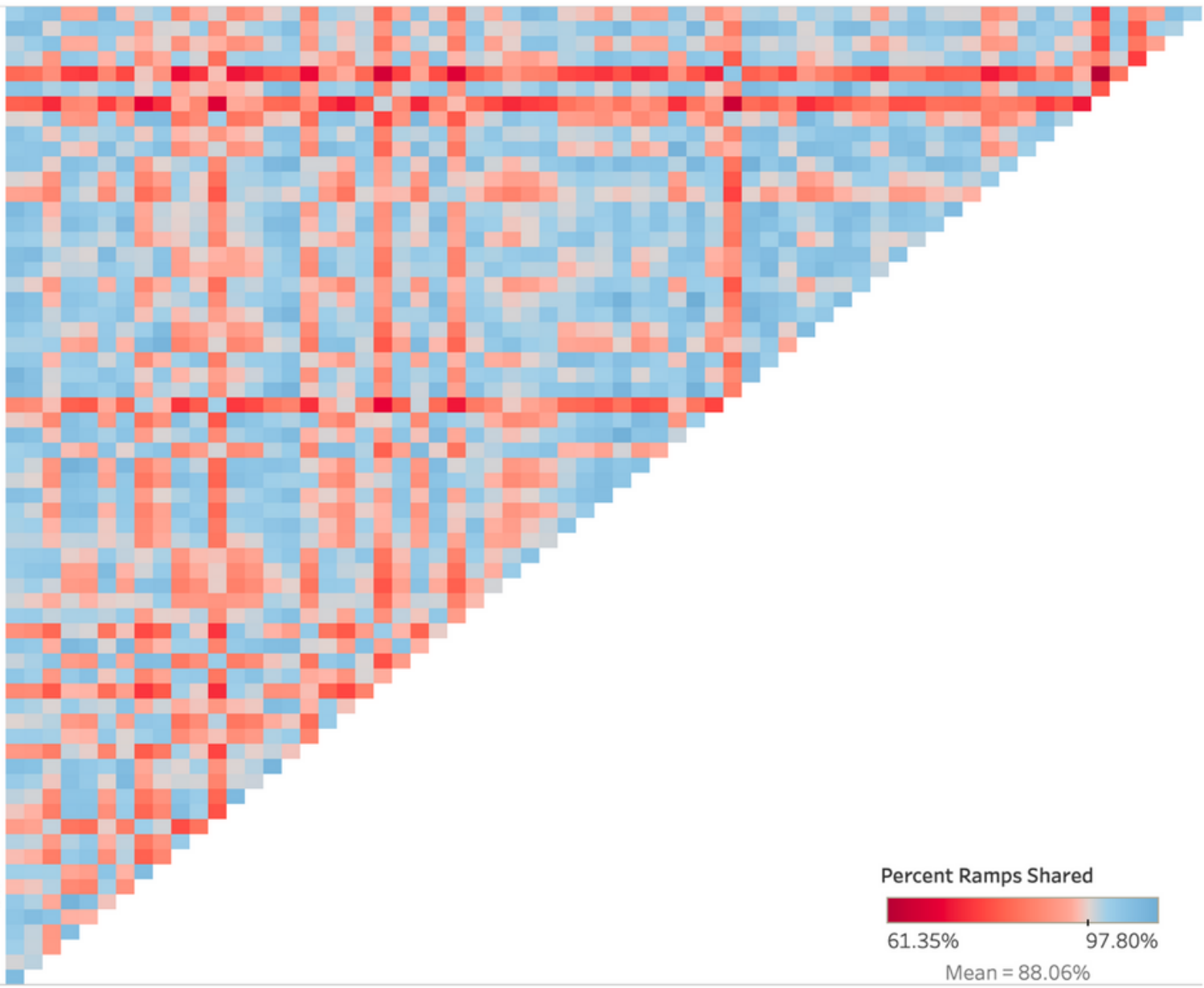

Figure 4

Heat Map of Percent of Ramp Sequences Shared between Tissue-Stratified Cell Types. The percent of ramp sequences present in one tissue-stratified cell type that are also present in another cell type. Red indicates fewer shared ramp sequences (i.e., more cell type-specific usage of ramp sequences). Tissuestratified cell type comparisons that are blue have more shared ramp sequences. 


\section{Percentage of Tissues with a Ramp Sequence in SARS-CoV-2}

\section{Genes}

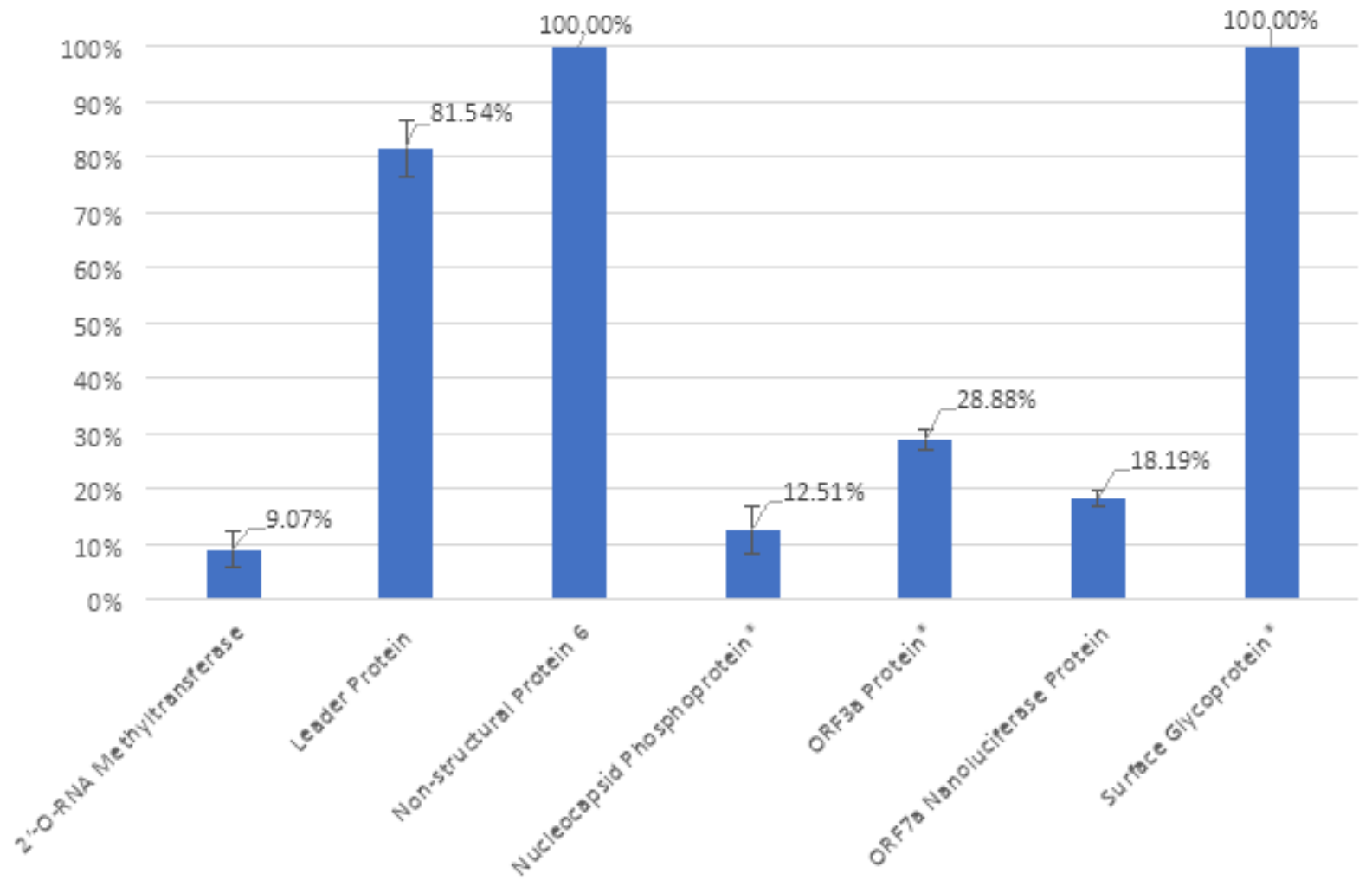

\section{Figure 5}

Percentage of Tissues with a Ramp Sequence in SARS-CoV-2 Genes. The percentage of 62 tissues in the consensus dataset in which seven SARS-CoV-2 genes have a ramp sequence. Error bars show the standard error between the four different datasets. In genes marked with an asterisk, less than one percent of all variants contained a ramp sequence. Notably, the other four genes had a ramp sequence in the one and only variant provided in the SARS-CoV-2 genome dataset. More exact data are available on the Ramp Atlas SARS-CoV-2 page: https://ramps.byu.edu/Covid. 


\section{Average Percentage of Tissues with a Ramp Sequence in}

SARS-CoV-2 Associated Human Genes

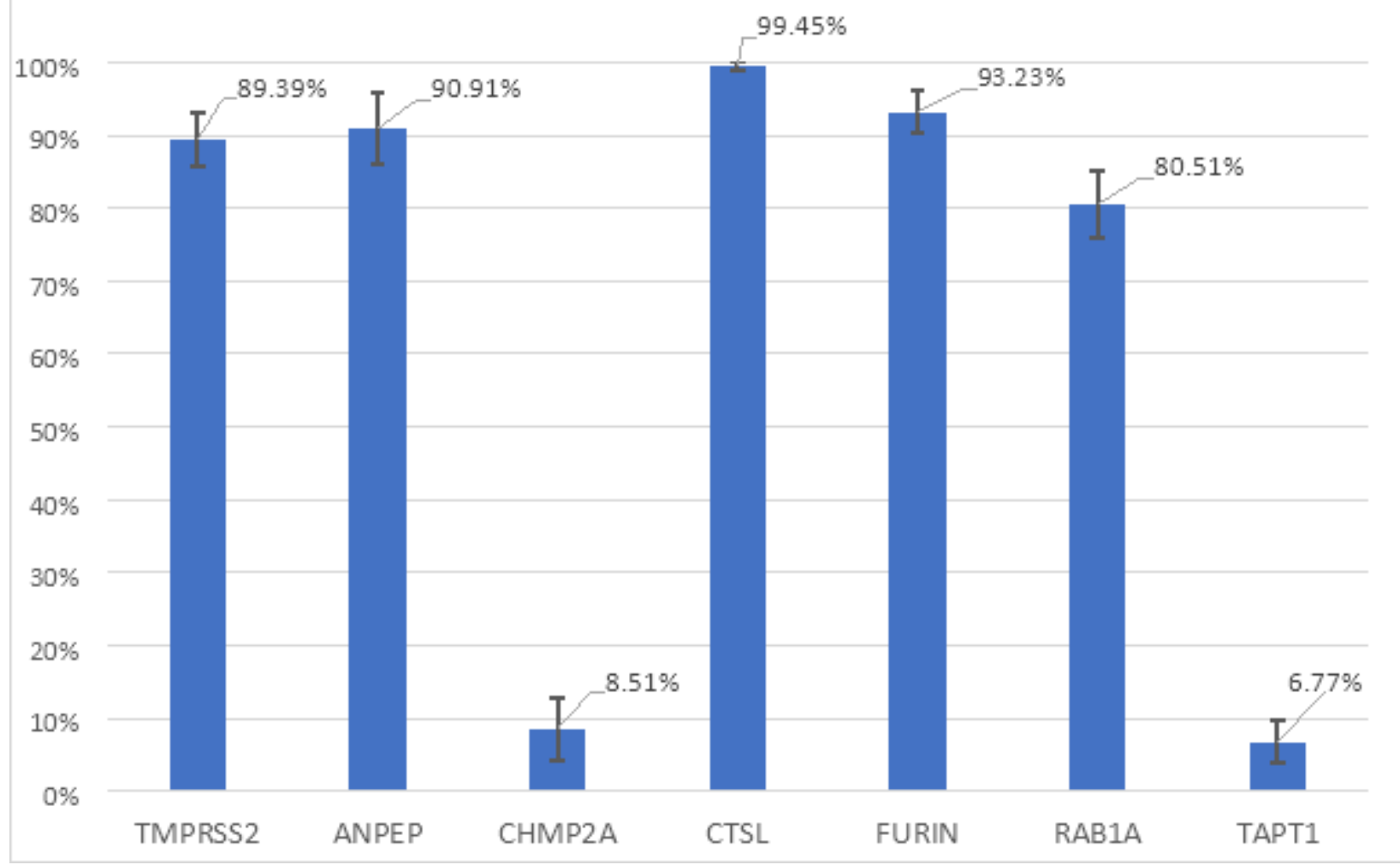

Figure 6

Percentage of Tissues with a Ramp Sequence in Human Genes for SARS-CoV-2 Entry Factors. The percentage of the 62 tissues in the consensus dataset in which human genes that code for SARS-CoV-2 entry factors have a ramp sequence. Error bars show the standard error between the four different datasets. 
Total Number of SARS-CoV-2 and Associated Human Entry Factor Genes with

a Ramp Sequence in 62 Human Tissues

14

12

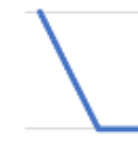

8

6

4

2

0

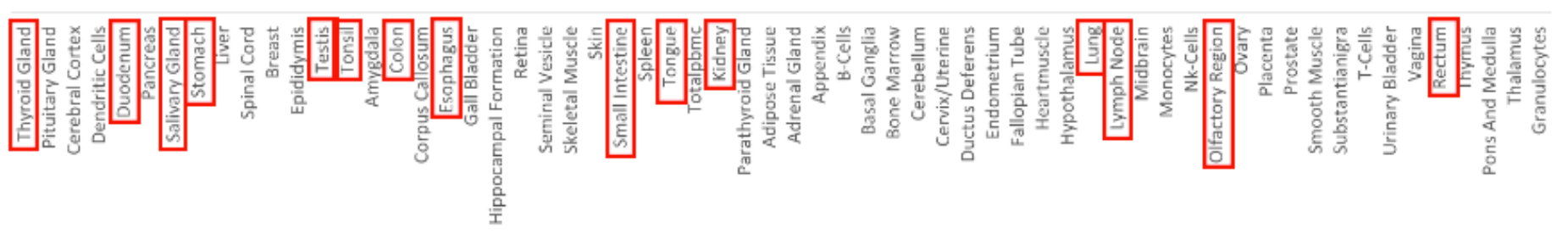

\section{Figure 7}

The Total Number of SARS-CoV-2 and Associated Human Entry Factor Genes with a Ramp Sequence in 62 Human Tissues. An aggregation of the data presented in Figures 5 and 6 , organized by tissue, and showing the total number of genes, both in the SARS-CoV-2 genome and associated human entry factors, with ramp sequences. Tissues where SARS-CoV-2 is found in higher amounts are marked in red boxes. These tissues had a significantly greater number of genes with ramp sequences ( $p$-value $=0.0099$ ). 

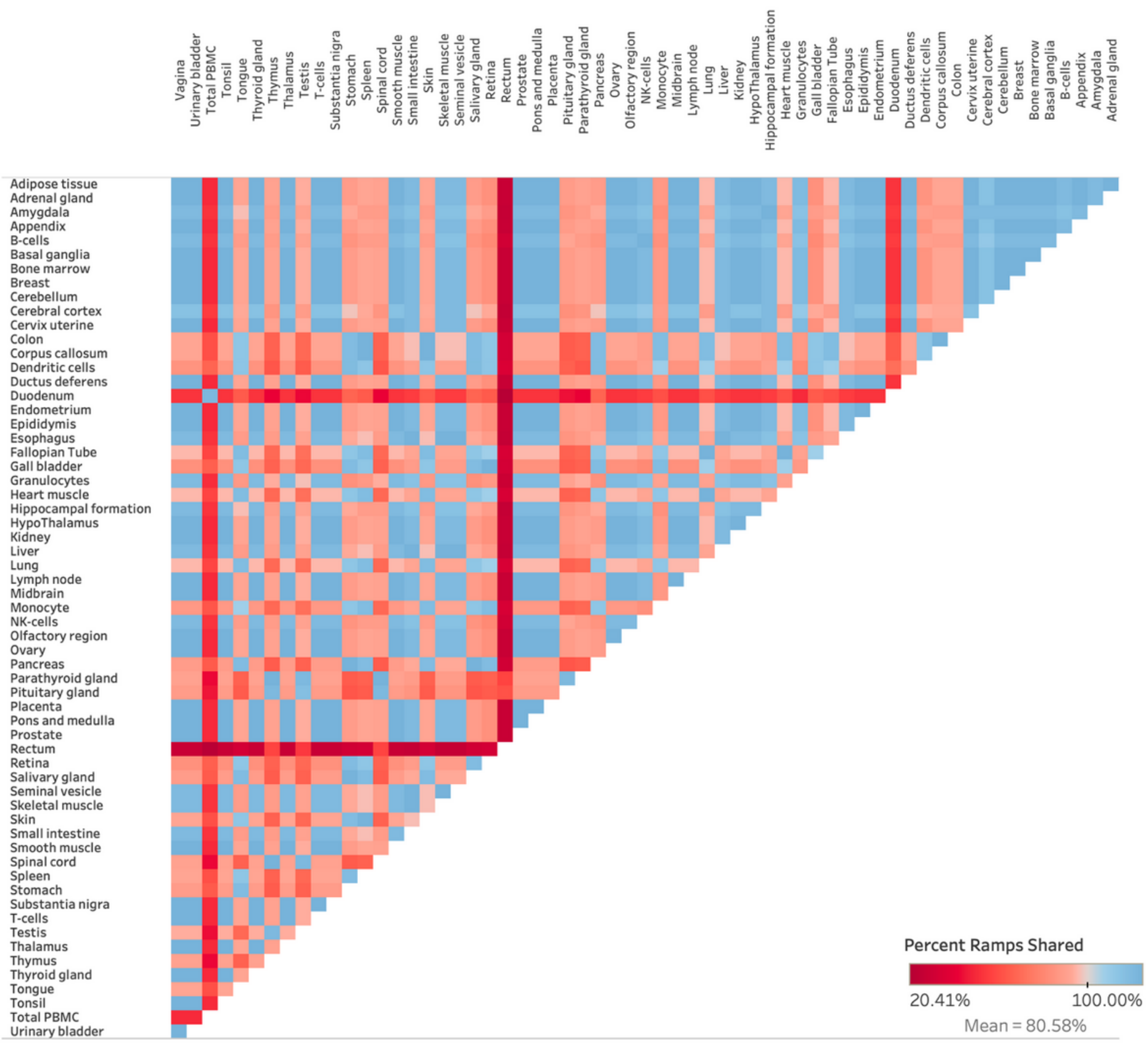

Figure 8

Heat Map of Percentage of SARS-CoV-2 Ramp Sequences Shared Between Tissues. The percentage of ramp sequences in SARS-CoV-2 genes that are present in both tissues being compared. Calculated using the consensus dataset. The redder the tissue comparison, the smaller the percentage of ramp sequences were present in both tissues. The bluer the comparison, the higher percentage of shared ramp sequences. 